\title{
FAST COMPUTATION OF MINIMAL ELEMENTARY DECOMPOSITIONS OF METABOLIC FLUX VECTORS
}

\author{
Raphael M. Jungers,Francisca Zamorano,Vincent D. Blondel, \\ Alain Vande Wouwer,Georges Bastin.
}

January 28,2010

\begin{abstract}
The concept of elementary flux vector is valuable in a number of applications of metabolic engineering. For instance, in metabolic flux analysis, each admissible flux vector can be expressed as a non-negative linear combination of a small number of elementary flux vectors. However a critical issue concerns the total number of elementary flux vectors which may be huge because it combinatorially increases with the size of the metabolic network. In this paper we present a fast algorithm that randomly computes a decomposition of admissible flux vectors in a minimal number of elementary flux modes without explicitly enumerating all of them. The method is illustrated with an experimental case-study on CHO cells where the network has 65329 elementary flux vectors while the admissible flux distributions are expressed as a combination of 22 elementary vectors only.
\end{abstract}

\section{1 introduction}

The intracellular metabolism of living cells is usually represented by a metabolic network under the form of a directed hypergraph that encodes a set of biochemical reactions taking place within the cell. In this hypergraph, the nodes represent the metabolites and the edges represent the metabolic fluxes.

According to the quasi steady-state paradigm of metabolic flux analysis (MFA) (e.g. [16]), it is assumed that the fluxes are balanced at each internal node. This means that the net sum of production and consumption fluxes, weighted by their stoichiometric coefficients, is zero for each internal metabolite of the network. This is expressed by the algebraic relation:

$$
\mathbf{N v}=\mathbf{0} \quad \mathbf{v} \geqslant \mathbf{0}
$$

where $\mathbf{v}=\left(v_{1}, v_{2}, \ldots, v_{m}\right)^{T}$ is the $m$-dimensional column vector of fluxes and $\mathbf{N}=\left[n_{i j}\right]$ is the $n \times m$ stoichiometric matrix of the metabolic network ( $m$ is the number of fluxes and $n$ the number of internal nodes of the network). More 
precisely, a flux $v_{j}$ denotes the rate of reaction $j$ and a non-zero $n_{i j}$ is the stoichiometric coefficient of the metabolite $i$ in reaction $j$.

For a given metabolic network, the set $S$ of possible flux distributions is the set of vectors $\mathbf{v}$ that satisfy the linear system (1). This set $S$ is the pointed polyhedral cone resulting from the intersection of the kernel of $\mathbf{N}$ with the nonnegative orthant. This implies that there exists a set of elementary flux vectors $\mathbf{e}_{i}([18]$ ) which are the edges (or extreme rays) of the polyhedral cone and such that any flux distribution $\mathbf{v}$ can be expressed as a non-negative linear combination of the vectors $\mathbf{e}_{i}$ which form therefore a unique convex basis (see e.g. [19]) of the flux space $S$ :

$$
\mathbf{v}=w_{1} \mathbf{e}_{1}+w_{2} \mathbf{e}_{2}+\cdots+w_{q} \mathbf{e}_{q} \quad w_{i} \geqslant 0 .
$$

From a biochemical viewpoint, the elementary flux vectors can be interpreted as the simplest metabolic pathways connecting extracellular substrates to final products. A typical application, among others, is when these vectors are used to translate the whole metabolic network into macroscopic bioreactions whose stoichiometric coefficients are given by the non-zero entries of the vectors $\mathbf{e}_{i}$ (see [16]). The term extreme pathways has also been used to denote the concept of extreme rays, even though the definitions slightly differ in technical aspects. These two notions have been largely compared in the literature (see e.g. [8, 11]).

The $m \times q$ non-negative matrix $\mathbf{E}$ with column vectors $\mathbf{e}_{i}$ obviously satisfies $\mathbf{N E}=0$ and (2) can be written in matrix form as

$$
\mathbf{v}=\mathbf{E w} \quad \text { with } \quad \mathbf{w} \triangleq\left(w_{1}, w_{2}, \ldots, w_{q}\right)^{T} .
$$

Thus, the elementary flux vectors are a way of representing the set of possible flux distributions. A well known issue related to this representation is that typically, the number of such vectors grows exponentially with the size of the problem. As an example, we study in Section 4 a relatively detailed metabolic network which involves 82 reactions and 53 metabolites. Though the dimension of this network does not seem so large, METATOOL calculates a number of elementary flux modes equal to 65329. Manipulating such a number of elementary flux vectors is not an easy task. For certain specific applications (e.g., recombinant protein production, media optimization, etc) more detailed metabolic networks involving several hundreds of reactions (e.g. [4]) can be of major interest. However, it is generally acknowledged that the enumeration of the elementary flux modes can already be out of reach for systems with more than $\approx 100$ reactions and metabolites ([17], see also [9]).

In this paper, we address the issue of decomposing a given flux distribution $\mathbf{v}$ in the convex basis of elementary flux vectors $\mathbf{e}_{i}$. In general, this decomposition does not necessitate the whole enumeration of the convex basis but requires only the knowledge of a few elementary vectors. The information needed for computing these elementary vectors can be obtained directly from the stoichiometric matrix $\mathbf{N}$ which is much smaller than the matrix $\mathbf{E}$.

Motivated by practical applications, including the analysis of metabolic pathways [12], the derivation of dynamic macroscopic models [13] and the char- 
acterization of metabolic phenotypes [4], our objective in this paper will precisely be to determine minimal such decompositions.

Well known theoretical results exist, that give upper bounds on the minimal number of vectors of the convex basis that are necessary to decompose the flux distribution. Namely, this number is $n-m$, which is typically very small in comparison with the number of elementary flux modes. As an example, in the case study of Section 4, the flux distribution can be expressed with 22 elementary flux modes, while the convex basis counts 65329 such vectors. We show how to compute this decomposition without actually computing the whole convex basis, thanks to convex programming techniques.

When the measurements do not determine the flux distribution in a unique way, the situation is more complex: it is possible to show that there is at least one compatible flux distribution for which even less elementary flux modes are necessary to decompose it. The problem is more complex in this case because it is not known a priori which vector, among all admissible flux distributions, is the one that can be decomposed in the minimal number of elementary flux modes. For this more difficult problem, we also provide a method that allows to compute this distribution in polynomial time, without computing the whole convex basis.

\section{Metabolic flux analysis and decomposition in elementary flux modes}

\subsection{Metabolic Flux Analysis}

Metabolic flux analysis (MFA) is the exercise of calculating the admissible flux distributions $\mathbf{v}$ that satisfy the steady state balance equation $\mathbf{N v}=\mathbf{0}$ together with an additional set of linear constraints added by using experimental measurements. Here we consider the case where the measurements are collected in a vector $\mathbf{v}_{m}$ which is a linear function of the unknown flux distribution $\mathbf{v}$ and is expressed as

$$
\mathbf{P} \mathbf{v}=\mathbf{v}_{m}
$$

where $\mathbf{P}$ is a given $p \times m$ full-rank matrix. In addition, we assume for the sake of clarity that $\mathbf{P e}_{i} \neq 0 \forall i$ or, in other terms, that the elementary flux vectors $\mathbf{e}_{i}$ do not belong to the kernel of the matrix $\mathbf{P}$. Then, from equations (1)-(4), we have the following fundamental equation of metabolic flux analysis

$$
\left(\begin{array}{c}
\mathbf{N} \\
\mathbf{P}
\end{array}\right) \mathbf{v}=\left(\begin{array}{c}
\mathbf{0} \\
\mathbf{v}_{m}
\end{array}\right) \quad \mathbf{v} \geqslant \mathbf{0}
$$

For a given metabolic network and a given set of measurements, the solution of the MFA problem is defined as the set $\mathcal{F}$ of admissible flux distributions, i.e. the set of non-negative vectors $\mathbf{v}$ that satisfy the linear system (5). Hence, as emphasized in [13, Chapter 4]-[14], the set $\mathcal{F}$ is a polytope in the positive orthant 


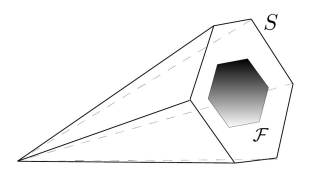

Figure 1: Illustration of the flux spaces $S$ and $\mathcal{F}$.

$\mathbb{R}_{+}^{m}$. This means that any admissible flux distribution $\mathbf{v}$ can be expressed as a convex combination of a set of non-negative basis vectors $\mathbf{f}_{i}$ which are the vertices of this polytope and form therefore a unique convex basis of the flux space $\mathcal{F}$. In other words, the solution of the MFA problem is the admissible flux space $\mathcal{F}$ defined as

$$
\mathcal{F} \triangleq\left\{\mathbf{v}: \mathbf{v}=\sum_{i} \alpha_{i} \mathbf{f}_{i}, \quad \alpha_{i} \geqslant 0\right\}
$$

The admissible flux space $\mathcal{F}$ is a subset of the possible flux space $S$. In geometric terms, the polytope $\mathcal{F}$ defines a subset of the pointed cone $S$ as illustrated in Fig.1.

\subsection{Minimal decomposition in elementary vectors}

For any admissible flux vector $\mathbf{v}$ in the polytope $\mathcal{F}$ satisfying equation (5), it must be emphasized that the decomposition of $\mathbf{v}$ in the convex basis $\left\{\mathbf{e}_{i}\right\}$ is not unique. As mentioned above, our aim is to determine minimal such decompositions.

Using (3), system (5) is equivalent to the system:

$$
\left(\begin{array}{c}
\mathbf{N E} \\
\mathbf{P E}
\end{array}\right) \mathbf{w}=\left(\begin{array}{c}
\mathbf{0} \\
\mathbf{v}_{m}
\end{array}\right) \quad \mathbf{w} \geqslant 0 .
$$

We observe that the first equation $\mathbf{N E w}=\mathbf{0}$ is trivially satisfied independently of $\mathbf{w}$ since by definition $\mathbf{N E}=0$. Hence, system (7) may be reduced to the second equation:

$$
\mathbf{P E w}=\mathbf{v}_{m} \quad \mathbf{w} \geqslant \mathbf{0} .
$$

In this form, it is clear that the set of admissible weighting vectors $\mathbf{w}$ that satisfy (8) again constitutes a convex polytope that we denote $\mathcal{H}$. Therefore there exists a set of appropriate edge vectors $\mathbf{h}_{i}$ such that any arbitrary convex combination of the form:

$$
\mathbf{w}=\sum_{i} \beta_{i} \mathbf{h}_{i} \quad \beta_{i} \geqslant 0 \quad \sum_{i} \beta_{i}=1
$$

is necessarily an admissible $\mathbf{w}$ satisfying (8). The convex basis vectors $\mathbf{h}_{i}$ have a critical property : the number of non-zero entries is equal to the size $p$ of the vector $\mathbf{v}_{\mathbf{m}}$, i.e. the number of measurements (see [5] and Section 3.5 in [13] for a proof). 
Theorem 1. [5] Any admissible flux distribution $\mathbf{v} \in \mathcal{F}$ can be expressed as a convex combination of $m-n$ elementary flux vectors $\mathbf{e}_{i}$.

Moreover, if $p<m-n$, then, there is at least one vector $\mathbf{v}^{*} \in \mathcal{F}$, such that $\mathbf{v}^{*}$ can be expressed as a convex combination of only $p$ elementary flux vectors.

From a metabolic viewpoint, each vector $\mathbf{h}_{i}$ is a solution $\mathbf{w}$ of (8) corresponding to a particular admissible flux distribution $\mathbf{v}$ :

$$
\mathbf{v}=\mathbf{E h}_{i} \quad \mathbf{v} \in \mathcal{F} .
$$

In this expression, the non-zero entries of the vector $\mathbf{h}_{i}$ are interpreted as the weights of the respective contributions of the corresponding elementary flux vectors $\mathbf{e}_{i}$ in the computation of the flux distribution $\mathbf{v}$.

An important issue concerns the number of distinct extreme rays or vertices that are generated when computing the cone $S$ or the polytopes $\mathcal{F}$ and $\mathcal{H}$. This number may become very large because it combinatorially increases with the size of the underlying metabolic network. More precisely, if $n$ is the number of metabolites and $m$ is the number of reactions, there is no polynomial function $p(m, n)$ such that the number of elementary flux modes $q$ satisfies $q \leq p(m, n)$ for all metabolic networks. It is also the case for the number of vectors $h_{i}$ that are vertices of the Polytope $\mathcal{H}$.

The Double Description (DD) method ([10]) is the simplest known algorithm for enumerating the extreme rays of a polyhedral cone (see [5] for a review). In the context of metabolic networks it has received various dedicated improvements that are documented in the literature (see e.g. [15], [6] and [7]). In practical applications of MFA, the enumeration of all extreme rays is not necessarily a critical objective. In many applications it is sufficient to know only one minimal decomposition of some vectors $\mathbf{v} \in \mathcal{F}$ in terms of elementary vectors $\mathbf{e}_{i}$. According to Theorem 1, there necessarily exist admissible $\mathbf{v}$ having a decomposition that involves only $p$ terms. Computing this decomposition may be very expensive at first sight since the dimension of $\mathbf{E}$ is not bounded by a polynomial in the sizes of $\mathbf{N}$ and $\mathbf{P}$.

In the next section we present a fast algorithm that randomly computes vectors $\mathbf{v} \in \mathcal{F}$ having such a minimal decomposition from the sole knowledge of the stoichiometric matrices $\mathbf{N}, \mathbf{P}$ and the measurement vector $\mathbf{v}_{m}$, but without explicitely enumerating the extreme rays of the cone $S$ (i.e. the columns of the huge matrix $\mathbf{E}$ ) and therefore without solving the system (8).

\section{The algorithm}

In this section we present efficient methods to decompose a flux distribution in a minimal number of elementary flux modes. We first comment on the term "minimal". We are interested here in finding a decomposition of the point $\mathbf{v}$ in $n-m$ elementary flux modes, or, if $p<n-m$, in $p$ elementary flux modes. That is, we are interested in matching the theoretical bounds of Theorem 1. This does 


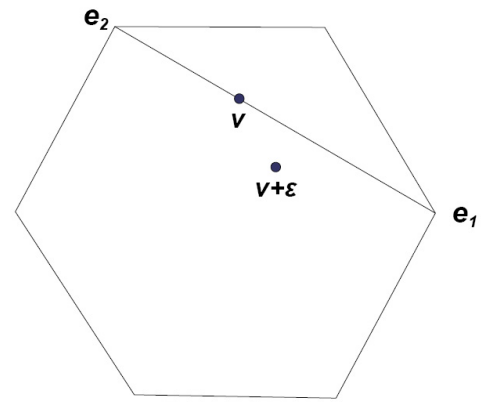

Figure 2: An example of a situation where the point $\mathbf{v}$ can be decomposed in even less vertices than the number provided by Theorem 1. However this phenomenon is sensitive to very small perturbations $\epsilon$.

not preclude the possibility that $\mathbf{v}$ could be expressed with even less elementary flux modes. This can be seen in Figure 2, where the point $\mathbf{v}$ is "by chance" exactly on a line passing through two vertices of the polytope. In this situation, while the theoretical bound tells us that $\mathbf{v}$ can be decomposed as a convex combination of 3 vertices, it actually appears that such a decomposition exists with 2 vertices. However, we are not interested in finding such decomposition for mainly two reasons: First, we are looking for a robust decomposition, while, as can be seen in Figure 2, a decomposition in less than 3 vertices is not robust: a slight perturbation $\epsilon$ of $\mathbf{v}$ can move it out of the line $\mathbf{e}_{1} \mathbf{e}_{2}$, so that the previous decomposition is not possible anymore. Since $\mathbf{v}_{m}$ is a vector of measurements (typically retrieved from a regression), it does not make sense to look for a decomposition which is sensitive to very small perturbations.

Second, this kind of minimal decomposition in even less vertices than theoretically foreseen is NP-hard to find, so that one cannot hope to develop a polynomial time algorithm to obtain such a decomposition [3].

Below, we first present a general algorithm that allows to decompose $\mathbf{v}$ in $m-n$ vertices. We then show how to reach the better decomposition in $p$ vertices in case $p<m-n$.

\subsection{Decomposing a vector in a convex basis}

Let us first consider the following problem: We are given a vector $\mathbf{v}$ that belongs to a cone $S$, and we would like to express this vector as a linear combination of a few extreme rays of $S$. The cone $S$ is given via its defining matrix $\mathbf{M}$.

The geometric intuition behind the algorithm can be understood in Figure 3 . We first pick randomly one vertex $\mathbf{e}_{1}$ of the cone $S$. This can be done easily, and we show below one method to do that. We then draw a line passing through this vertex and the point $\mathbf{v}$. This line hits the boundary of the polytope at two points: $\mathbf{e}_{1}$, and $\mathbf{v}_{1}$, which belongs to a face $\mathcal{P}_{1}$ of $S$. So, $\mathbf{v}$ can be expressed as a 


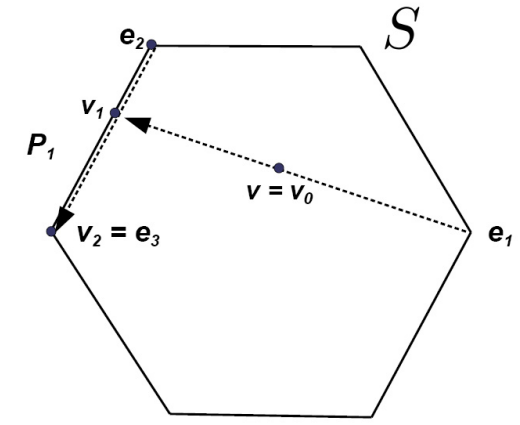

Figure 3: Decomposition of $\mathbf{v}$ in the convex basis $\left\{e_{i}\right\}$.

convex combination of $\mathbf{e}_{1}$ and $\mathbf{v}_{1}$. Now we proceed iteratively, by decomposing $\mathbf{v}_{i}$ as a convex combination of vertices of $\mathcal{P}_{i}$ (and then of $S$ ). The process ends after at most $k$ steps, when $\mathcal{P}_{i}$ is a single point $\mathbf{v}_{i}$, thus being also a vertex of $S$. All the optimization problems we have to solve in order to do that can be cast as linear programs and can then be solved in polynomial time with classical Linear Programming methods (e.g. [2]).

We now present the algorithm with its technical details. Let us denote $a=\mathbf{1}^{T} \mathbf{v}$ the sum of the entries in $\mathbf{v}$ ( $\mathbf{1}$ denotes the vector whose all entries are equal to one). In the following we will consider without loss of generality the slightly different problem where we are looking for extreme rays $\mathbf{e}_{i}$ such that $\mathbf{1}^{T} \mathbf{e}_{i}=a$. Geometrically speaking, we cut the cone with a plane passing through $\mathbf{v}$ such that the intersection is a bounded polytope whose vertices correspond to extreme rays of the initial cone $S$. We are thus given a (bounded) polytope $\mathcal{P}$, and a vector $\mathbf{v}$ in this polytope and we want to express this vector $\mathbf{v}$ as a convex combination of vertices of the polytope.

We first pick up (at random) a vertex of the polytope $\mathcal{P}$. The problem of finding a vertex of the polytope defined by the equations

$$
\mathbf{M x}=\mathbf{0}, \quad \mathbf{1}^{T} \mathbf{x}=a, \quad \mathbf{x} \geqslant \mathbf{0}
$$

can be solved in time polynomial in the number of constraints and the dimension. Indeed, consider the following linear program :

$$
\begin{aligned}
& \min \mathbf{d}^{T} \mathbf{x} \\
& \text { s.t. } \\
& \mathbf{M} \mathbf{x}=\mathbf{0}, \\
& \mathbf{x} \geqslant \mathbf{0}, \\
& \mathbf{1}^{T} \mathbf{x}=a .
\end{aligned}
$$

If $\mathbf{d}$ is not parallel to a constraint of the program (11), then, the solution is a vertex of the corresponding polytope. So in practice, if $\mathbf{d}$ is a random direction, an extreme ray is found with probability one. 
We then iterate, by projecting $\mathbf{v}$ on faces $\mathcal{P}_{i}$ of the polytope $\mathcal{P}$ described by the constraints of the program (11). Since the dimension of the face $\mathcal{P}_{i}$ strictly decreases at each step, the algorithm takes at most $k-1$ steps, where $k$ is the dimension of the polytope $\mathcal{P}$.

Take any vertex $\mathbf{e}_{1}$ of $\mathcal{P}$ (for instance by solving the linear program (11)); then the vector $\mathbf{v}$ can be written as the convex combination of $\mathbf{e}_{1}$ and of a vector $\mathbf{v}_{1}$, which belongs to a face $\mathcal{P}_{1}$ of $\mathcal{P}: \mathbf{v}=\gamma_{1} \mathbf{e}_{1}+\left(1-\gamma_{1}\right) \mathbf{v}_{1}$ (see Figure 3 ). These quantities $\mathbf{v}_{i}, \gamma_{i}$ are easy to compute, as $\mathbf{v}_{1}$ is the solution $\mathbf{x}^{*}$ of the Linear Program

$$
\begin{aligned}
& \max \mu \\
& \text { s.t. } \\
& \mathbf{M} \mathbf{x}=\mathbf{0}, \\
& \mathbf{x} \geqslant \mathbf{0}, \\
& \mathbf{1}^{T} \mathbf{x}=a, \\
& \mathbf{v}+\mu\left(\mathbf{v}-\mathbf{e}_{1}\right)=\mathbf{x} .
\end{aligned}
$$

The geometric meaning of this linear program is as follows: starting from the vector $\mathbf{v}$ one tries to find a point $\mathbf{x}$ which is diametrically opposite to $\mathbf{e}_{1}$ and as far as possible from $\mathbf{v}$, and $\mu$ represents the distance from $\mathbf{v}$ to $\mathbf{x}$. Clearly this point will be on a face $\mathcal{P}_{1}$ of the polytope.

Now, at each step $i=1, \ldots, k^{\prime}, \mathcal{P}_{i}$ is a new polytope, and, since $\mathbf{v}_{i} \in \mathcal{P}_{i}$, we still can express $\mathbf{v}_{i}$ as a convex combination of a vertex of $\mathcal{P}_{i}$ (which is also a vertex of $\mathcal{P}$ ) and a point $\mathbf{v}_{i+1}$ that belongs to a face $\mathcal{P}_{i+1}$ of $\mathcal{P}_{i}$ (which is also a face of $\mathcal{P}$, but of dimension strictly smaller than $\operatorname{dim} \mathcal{P}_{i}$ ). Thus, after $k^{\prime} \leq k$ steps, the dimension of $\mathcal{P}_{k^{\prime}}$ is equal to 0 , which means that $\mathbf{v}_{k^{\prime}}$ is actually a vertex of $\mathcal{P}$ which we denote $\mathbf{e}_{k^{\prime}+1}$. Thus, $\mathbf{v}_{k^{\prime}-1}=\gamma_{k^{\prime}} \mathbf{e}_{k^{\prime}}+\left(1-\gamma_{k^{\prime}}\right) \mathbf{e}_{k^{\prime}+1}$. Finally we can write:

$$
\begin{aligned}
\mathbf{v} & =\mathbf{v}_{0} \\
& =\gamma_{1} \mathbf{e}_{1}+\left(1-\gamma_{1}\right)\left(\gamma_{2} \mathbf{e}_{2}+\left(1-\gamma_{2}\right)\left(\ldots \left(\gamma_{k^{\prime}} \mathbf{e}_{k^{\prime}}+\right.\right.\right. \\
& \left.\left.\left.\left(1-\gamma_{k^{\prime}}\right) \mathbf{e}_{k^{\prime}+1}\right)\right)\right) \\
& =\sum_{1}^{k^{\prime}+1} w_{i} \mathbf{e}_{i}
\end{aligned}
$$

with $\sum w_{i}=1$.

Thus, since the dimension of the cone $S$ is equal to $k+1=m-n$, we obtain at most $m-n$ extreme vectors $\mathbf{e}_{i}$. We have thus found the decomposition in polynomial time, which is a dramatic improvement compared to the naive brute force approach that requires the enumeration of all vectors $\mathbf{e}_{i}$.

\subsection{Finding the minimal decomposition if $p<m-n$}

We now suppose that $p<m-n$, which implies that there are several vectors $\mathbf{v}$ that satisfy Equation (5) (the system is underdetermined). By Theorem 1, 
we know that at least one of them can be expressed as a convex combination of only $p$ vertices of $S^{1}$. In other words, there are admissible vectors w (the extreme rays of $\mathcal{H}$ ), that only contain at most $p$ nonzero values. However, if one does not want to compute the matrix $\mathbf{E}$ of extreme rays of $S$, this is not an easy task a priori to find such a minimal representation. Indeed, the dimension of $\mathbf{w}$ is exponential in the size of the problem. Thus, we are facing a more complex problem, since we want to decompose a point as a convex combination of vertices of the cone, but we even do not know a priori which is this point, among the whole set of points that satisfy Equation (5).

In order to compute such a "good" vector $\mathbf{v}$ and its corresponding decomposition, we introduce yet another cone $\mathcal{K} \subset \mathbb{R}^{p}$. This cone is the projection of $S$ by the matrix $\mathbf{P}$ :

$$
\mathcal{K}=\{\mathbf{y}=\mathbf{P} \mathbf{v}: \mathbf{v} \geqslant \mathbf{0}, \mathbf{N v}=\mathbf{0}\}
$$

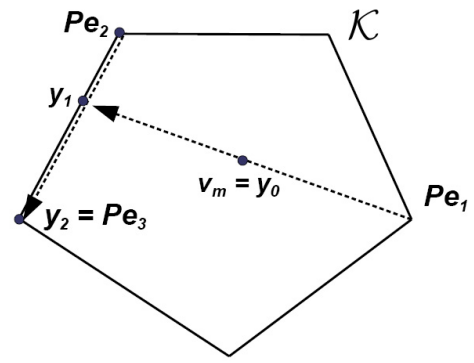

Figure 4: Decomposition of $\mathbf{v}$ in the case where $p<m-n$. The algorithm actually decomposes the vector $\mathbf{v}_{\mathbf{m}}$ in a "small" $p$-dimensional cone, which is the projection of $\mathcal{S}$ under the matrix $P$.

The idea of the algorithm is as follows (see Figure 4): We know that the vector $\mathbf{v}_{m}$ is in $\mathcal{K}$ (see Equation (5)). So, it can be expressed as a convex combination of $p$ extreme rays of $\mathcal{K}$ (because $\mathcal{K}$ has dimension $p$ ). Now, the extreme rays of $\mathcal{K}$ are the projections of extreme rays $\mathbf{e}_{i}$ of $\mathcal{S}$ under the matrix $\mathbf{P}$. This implies that the corresponding convex combination of the $\mathbf{e}_{i}$ gives us the required $\mathbf{v}$. We start from an extreme ray $\mathbf{e}_{1}$ of the cone $\mathcal{S}$ (for instance by applying the Linear Program (11)); then the vector $\mathbf{v}_{m}=\mathbf{y}_{0}$ can be written as the convex combination of $\mathbf{P e}_{1}$ and a vector $\mathbf{y}_{1}$, which belongs to a face $\mathcal{P}_{1}$ of $\mathcal{K}: \mathbf{v}_{m}=\alpha_{1} \mathbf{P e}_{1}+\left(1-\alpha_{1}\right) \mathbf{y}_{1}$. This vector $\mathbf{v}_{1}$ is easy to find with a line search in the cone $\mathcal{K}$ as in Program (12). Now, at each step, find an extreme ray $\mathbf{e}_{i}$ of $\mathcal{K}$ which is mapped to the face $\mathcal{P}_{i-1}$ of $\mathcal{K}$. Then $\mathbf{y}_{i-1}$ can be expressed as a convex combination of $\mathbf{P e}_{i}$ and a vector $\mathbf{y}_{i}$ that belongs to a face $\mathcal{P}_{i}$ of $\mathcal{P}_{i-1}$.

\footnotetext{
${ }^{1}$ To see this, consider the expression (8) of the polytope $\mathcal{H}$, which describes the set of admissible values of $\mathbf{w}$. It can be defined by only $p$ equalities, so that $\operatorname{dim}(\mathbf{w})-p$ inequality constraints can be activated to define an extreme ray $\mathbf{h}_{i}$ of $\mathcal{H}$.
} 


\begin{tabular}{|c|c|c|c|}
\hline Glucose & $-0,187130$ & Glutamine & $-0,050246$ \\
\hline Threonine & $-0,001184$ & Lysine & $-0,002125$ \\
\hline Valine & $-0,001956$ & Isoleucine & $-0,001528$ \\
\hline Leucine & $-0,002601$ & Phenylalanine & $-0,000998$ \\
\hline Methionine & $-0,000724$ & Asparagine & $-0,001278$ \\
\hline Arginine & $-0,002142$ & Proline & $-0,002142$ \\
\hline Histidine & $-0,003298$ & Tyrosine & $-0,007610$ \\
\hline Aspartate & $-0,000318$ & Cysteine & $-0,000923$ \\
\hline Glycine & 0,002230 & Serine & $-0,000923$ \\
\hline Glutamate & $-0,009548$ & Ammonia & 0,045712 \\
\hline Lactate & 0,344510 & Alanine & 0,008808 \\
\hline
\end{tabular}

Table 1: Vector of measurements $\mathbf{v}_{m}\left(\mathrm{mM} /\left(\mathrm{h} \times 10^{9}\right.\right.$ cells $\left.)\right)$, with a "-" sign for degradation.

Since the dimension of $\mathcal{P}_{i}$ strictly decreases at each step, after $t \leq p-1$ steps the point $\mathbf{y}_{t}$ is actually an extreme ray of $\mathcal{K}$, and is thus the projection of an extreme ray $\mathbf{e}_{(t+1)}$ of $S$. Finally we have the relations:

$$
\mathbf{v}_{m}=\sum_{1}^{t+1} \lambda_{i} \mathbf{P} \mathbf{e}_{i}=\mathbf{P}\left(\sum_{1}^{t+1} \lambda_{i} \mathbf{e}_{i}\right),
$$

and thus the vector

$$
\mathbf{v}=\sum_{1}^{t+1} \lambda_{i} \mathbf{e}_{i}
$$

is a convex combination of at most $p$ extreme vectors of $S$ that satisfies (5).

\section{Case study}

As a matter of illustration and motivation to the methodology presented above, we consider the example of chinese hamster ovary $(\mathrm{CHO})$ cells cultivated in batch mode in stirred flasks in a serum-free medium ([1]). During the growth phase, we assume that the cell metabolism is described by the metabolic network presented in Appendix A. The network involves the Glycolysis pathway, the Pentose-Phosphate pathway, the Krebs cycle, the amino-acid metabolism, the urea cycle as well as the nucleotide, protein and lipid synthesis (see [20] for further motivation and details).

For this network we have $m=82$ fluxes and $n=53$ internal metabolites (i.e. $m-n=29$ ), and there are 65329 elementary flux vectors $\mathbf{e}_{i}$ (i.e. the polyhedral cone $S$ has 65329 edges).

Moreover, there are $p=22$ extra-cellular species whose degradation or accumulation rates in the culture medium are measured and collected in the vector $\mathbf{v}_{m}$ given in Table 1. 
The algorithm of Section 4 is then implemented with these data. We present a trial where the resulting admissible flux vector $\mathbf{v}$ is given in Table 2 . It can be checked to satisfy (5) and to be fully consistent with the experimental data of Table 1. Furthermore, the algorithm provides the minimal decomposition of $\mathbf{v}$ as a non-negative linear combination of the 22 elementary flux vectors $\mathbf{e}_{i}$ given in Tables $3,4,5$.

Let us insist that the obtained vector $\mathbf{v}$ is obviously just one possible solution among many others with a minimal decomposition. If the algorithm is re-run with the same initial data, it will find other solutions with a minimal decomposition because it makes use of random searching directions. Complementary results on the metabolic flux analysis of CHO cells can be found in [20].

\section{Conclusions and perspectives}

The computation of a convex basis corresponding to a metabolic reaction network takes a huge amount of (time and space) computing resources. This cost can in some sense not be avoided, since in general the convex basis of a set of reactions grows exponentially with the number of reactions and metabolites. As reported by [9], already with $\approx 100$ reactions and metabolites, the number of vectors in the convex basis can rule out its explicit computation. Moreover, much larger networks are increasingly considered in the literature (for instance reference [4] deals with a network having 1175 fluxes and 733 internal metabolites, see also the BIGG database ${ }^{2}$ ) so that we can expect that in the future new methods to analyze bioreaction networks, like the one proposed in this paper, will be needed.

The goal of this paper was to show that even though the enumeration of the convex basis can be prohibitive, this does not preclude to process this convex basis in some implicit way, so that much important information about the system may be retrieved without explicitely and extensively computing the convex basis. We exemplify this in the present paper with the decomposition of the flux distribution in a minimal number of elementary flux modes, and it can be expected that many more issues on elementary flux modes will be addressed in the future by processing the convex basis in an implicit way rather than by giving an exhaustive list of its components.

Linear Programming techniques may provide efficient ways to analyze bioreaction and metabolic networks. In this paper we have used only a small part of the power of these techniques. For instance, the choice of the elementary flux modes in our algorithm is done at each step at random, while one could imagine to add an objective function to optimize the decomposition with regard to a certain objective.

\footnotetext{
${ }^{2}$ http://bigg.ucsd.edu/
} 


\section{6 acknowledgement}

This research is supported by the Scientific Network DYSCO (Dynamical Systems, Control, and Optimization), funded by the Belgian Programme on Interuniversity Attraction Poles (IAP VI/4). R. Jungers is a FNRS fellow and a BAEF fellow.

A matlab implementation of our algorithms is available on

http://www.inma.ucl.ac.be/ jungers/contents/efm.zip.

\section{References}

[1] J.S. Ballez, J. Mols, J. Burteau, S.N. Agathos, and Y-J. Schneider. Plant protein hydrolysates support CHO-320 cells proliferation and recombinant ifn-gamma production in suspension and inside microcarriers in protein-free media. Cytotechnology, 44:103-114, 2004.

[2] S. Boyd and L. Vandenberghe. Convex Optimization. Cambridge University Press, New-York, USA, 2004.

[3] D. Donoho and J. Tanner. Sparse nonnegative solution of underdetermined linear equations by linear programming. Proceedings of the National Academy of Sciences, 102(27), 2005.

[4] I. Famili, J. Forster, J. Nielsen, and B. O Palsson. Saccharomyces cerevisiae phenotypes can be predicted by using constraint-based analysis of a genome-scale reconstructed metabolic network. Proceedings of the National Academy of Sciences of the USA, 100(23):13134-13139, 2003.

[5] K. Fukuda and A. Prodon. The double description method revisited. In R. Euler and M. E. Deza I. Manoussakis, editors, Combinatorics and Computer Science, volume 1120 of Lecture Notes in Computation Sciences, pages 91-111. Springer-Verlag, 1996.

[6] J. Gagneur and S. Klamt. Computation of elementary modes : a unifying framework and the new binary approach. BMC Bioinformatics, 5:175, 2004.

[7] S. Klamt, J. Gagneur, and A. von Kamp. Algorithmic approaches for computing elementary modes in large biochemical networks. IEE Proceedings Systems Biology, 152:249-255, 2005.

[8] S. Klamt and J. Stelling. Two approaches for metabolic pathway analysis? Trends Biotechnol, 21(2):64-69, 2003.

[9] S. Klamt and J. Stelling. Stoichiometric and constraint-based modeling. In System Modeling in Cellular Biology, pages 73-96. 2006.

[10] T.S. Motzkin, H. Raiffa, G.L. Thompson, and R.M. Thrall. The double description method. In H.W. Kuhn and A.W. Tucker, editors, Contribution 
to the Theory of Games Vol. II, volume 28 of Annals of Mathematical Studies, pages 51-73, Princeton, New Jersey, 1953. Princeton University Press.

[11] J. A. Papin, J. Stelling, N. D. Price, S. Klamt, S. Schusterand, and B. O. Palsson. Comparison of network-based pathway analysis methods. Trends Biotechnol, 22(8):400-405, 2004.

[12] N.D. Price, J.A. Papin, and B.O. Palsson. Determination of redundancy and systems properties in the metabolic network of helicobacter pylori using genome-scale extreme pathway analysis. Genome Research, pages 760-769, 2002 .

[13] A. Provost. Metabolic design of dynamic bioreaction models. PhD thesis, Faculty of Engineering, Université Catholique de Louvain, November 2006.

[14] A. Provost and G. Bastin. Metabolic flux analysis : an approach for solving non-stationary underdetermined systems. In CD-Rom Proceedings 5th MATHMOD Conference, Paper 207 in Session SP33, Vienna, Austria, 2006.

[15] R. Schuster and S. Schuster. Refined algoritm and computer program for calculating all non-negative fluxes admissible in steady states of biochemical reaction systems with or without some flux rates fixed. Computer Applications in the Biosciences, 9(1):79-85, February 1993.

[16] G. Stephanopoulos, J. Nielsen, and A. Aristidou. Metabolic Engineering : Principles and Methodologies. Academic Press, San Diego, 1998.

[17] Marco Terzer and Jörg Stelling. Accelerating the computation of elementary modes using pattern trees. In WABI, pages 333-343, 2006.

[18] R. Urbanczik. Enumerating constrained elementary flux vectors of metabolic networks. IET Systems Biology, 1(5):274-279, 2007.

[19] H. Weyl. The elementary theory of convex polyhedra. In Contributions to the Theory of Games Vol. I, Annals of Mathematical Studies, pages 3-18, Princeton, New Jersey, 1950. Princeton University Press.

[20] F. Zamorano, A. Vande Wouwer, and G. Bastin. A detailed metabolic flux analysis of an underdetermined network of cho cells. Submitted to Journal of Biotechnology, 2009.

\section{A Metabolic network.}

Glycolisis

$\mathrm{v} 1: \mathrm{Glu}+\mathrm{ATP} \rightarrow \mathrm{G} 6 \mathrm{P}+\mathrm{ADP}$

$\mathrm{v} 2: \mathrm{G} 6 \mathrm{P}+\mathrm{ATP} \rightarrow \mathrm{DHAP}+\mathrm{G} 3 \mathrm{P}+\mathrm{ADP}$

v3: $\mathrm{DHAP} \rightarrow \mathrm{G} 3 \mathrm{P}$

$\mathrm{v} 4: \mathrm{G} 3 \mathrm{P}+\mathrm{OxP}+\mathrm{P}_{i}+\mathrm{ADP} \rightarrow(3) \mathrm{PG}+\mathrm{RdP}+\mathrm{ATP}$ 
v5: (3)PG $+\mathrm{ADP} \rightarrow \mathrm{Pyr}+\mathrm{ATP}$

Krebs Cycle

$\mathrm{v} 6: \mathrm{Pyr}+\mathrm{OxP} \rightarrow \mathrm{AcCoA}+\mathrm{CO}_{2}+\mathrm{RdP}$

v7: AcCoA + Oxal $\rightarrow$ Cit

v8:Cit $+\mathrm{OxP} \rightarrow \alpha \mathrm{KG}+\mathrm{CO}_{2}+\mathrm{RdP}$

$\mathrm{v} 9: \alpha \mathrm{KG}+\mathrm{OxP} \rightarrow \mathrm{SucCoA}+\mathrm{CO}_{2}+\mathrm{RdP}$

$\mathrm{v} 10: \mathrm{SucCoA}+\mathrm{ADP}+\mathrm{P}_{i} \rightarrow$ Succ $+\mathrm{ATP}$

v11:Succ $\rightarrow$ Fum

v12:Fum $\rightarrow$ Mal

$\mathrm{v} 13: \mathrm{Mal}+\mathrm{OxP} \rightarrow \mathrm{Oxal}+\mathrm{RdP}$

Pyruvate Fates

$\mathrm{v} 14: \mathrm{Pyr}+\mathrm{RdP} \rightarrow$ Lact $+\mathrm{OxP}$

$\mathrm{v} 15: \mathrm{Pyr}+\mathrm{Glu} \rightarrow \mathrm{Ala}+\alpha \mathrm{KG}$

Pentose Phosphate Pathway

v16: $\mathrm{G} 6 \mathrm{P}+2 \mathrm{OxP} \rightarrow \mathrm{R} 5 \mathrm{P}+2 \mathrm{RdP}+\mathrm{CO}_{2}$

v17: $3 \mathrm{R} 5 \mathrm{P} \rightarrow 2.5 \mathrm{G} 6 \mathrm{P}+0.5 \mathrm{P}_{i}$

Anaplerotic Reaction

v18: $\mathrm{Mal}+\mathrm{OxP} \rightarrow \mathrm{Pyr}+\mathrm{CO}_{2}+\mathrm{RdP}$

Amino Acid Metabolism

$\mathrm{v} 19: \mathrm{Glu}+\mathrm{OxP} \rightarrow \alpha \mathrm{KG}+\mathrm{NH}_{4}^{+}+\mathrm{RdP}$

$\mathrm{v} 20: \mathrm{Oxal}+\mathrm{Glu} \rightarrow \mathrm{Asp}+\alpha \mathrm{KG}$

v21: $\mathrm{Gln} \rightarrow \mathrm{Glu}+\mathrm{NH}_{4}^{+}$

$\mathrm{v} 22: \mathrm{Thr}+\mathrm{OxP} \rightarrow \mathrm{Gly}+\mathrm{AcCoA}+\mathrm{RdP}$

v23: $\mathrm{Gly}+\mathrm{OxP} \rightarrow \mathrm{CO}_{2}+\mathrm{NH}_{4}^{+}+\mathrm{RdP}$

v24: $(3) \mathrm{PG}+\mathrm{OxP}+\mathrm{Glu} \rightarrow \mathrm{Ser}+\alpha \mathrm{KG}+\mathrm{RdP}+\mathrm{P}_{i}$

v25: Ser $\rightarrow$ Gly

v26: $\mathrm{Ser} \rightarrow \mathrm{Pyr}+\mathrm{NH}_{4}^{+}$

v27: $\mathrm{Thr} \rightarrow \alpha \mathrm{Kb}+\mathrm{NH}_{4}^{+}$

v28: $\alpha \mathrm{Kb}+\mathrm{OxP} \rightarrow$ PropCoA $+\mathrm{RdP}+\mathrm{CO}_{2}$

v29: $\mathrm{PropCoA}+\mathrm{CO}_{2}+\mathrm{ATP} \rightarrow \mathrm{SucCoA}+\mathrm{ADP}+\mathrm{P}_{i}$

v30: $\mathrm{Lys}+2 \alpha \mathrm{KG}+\mathrm{OxP} \rightarrow \alpha \mathrm{Ka}+2 \mathrm{Glu}+\mathrm{RdP}$ v31: $\alpha \mathrm{Ka}+2 \mathrm{OxP} \rightarrow$

AcetoAcCoA $+2 \mathrm{RdP}+2 \mathrm{CO}_{2}$

v32: AcetoAcCoA $\rightarrow 2 \mathrm{AcCoA}$

v33: $\mathrm{Val}+\alpha \mathrm{KG} \rightarrow \alpha \mathrm{Kv}+\mathrm{Glu}$

v34: $\alpha \mathrm{Kv}+3 \mathrm{OxP} \rightarrow$ PropCoA $+2 \mathrm{CO}_{2}+3 \mathrm{RdP}$

v35: Ile $+\alpha \mathrm{KG} \rightarrow(3) \operatorname{Methyl}(2)$ oxovalerate $+\mathrm{Glu}$

v36: (3)Methyl (2)oxovalerate $+2 \mathrm{OxP} \rightarrow \mathrm{AcCoA}+\mathrm{PropCoA}+\mathrm{CO}_{2}+2 \mathrm{RdP}$

v37: $\mathrm{Leu}+\alpha \mathrm{KG} \rightarrow \alpha \mathrm{Ki}+\mathrm{Glu}$

v38: $\alpha \mathrm{Ki}+\mathrm{OxP}+\mathrm{ATP} \rightarrow \mathrm{AcCoA}+\mathrm{AcetoAc}+\mathrm{RdP}+\mathrm{ADP}+\mathrm{P}_{i}$

v39: AcetoAc $+\mathrm{SucCoA} \rightarrow$ AcetoAcCoA + Succ

v40: $\mathrm{Phe}+\mathrm{RdP} \rightarrow \mathrm{Tyr}+\mathrm{OxP}$

$\mathrm{v} 41: \mathrm{Tyr}+\alpha \mathrm{KG} \rightarrow \mathrm{Fum}+\mathrm{Glu}+\mathrm{AcetoAc}+\mathrm{CO}_{2}$ 
v42: Met $+\mathrm{ATP} \rightarrow$ HomoCys $+\mathrm{AMP}+\mathrm{P}_{i}$

v43: HomoCys $+\mathrm{Ser} \rightarrow \alpha \mathrm{Kb}+\mathrm{Cys}+\mathrm{NH}_{4}^{+}$

v44: $\mathrm{Cys} \rightarrow \mathrm{Pyr}+\mathrm{NH}_{4}^{+}$

v45: Asn $\rightarrow$ Asp $+\mathrm{NH}_{4}^{+}$

v46: Arg $\rightarrow$ Ornitine + Urea

v47: Ornitine $+\alpha \mathrm{KG} \rightarrow$ Glu $\gamma \mathrm{SA}+\mathrm{Glu}$

v48: Pro $\rightarrow$ Glu $\gamma$ SA

v49: Glu $\gamma \mathrm{SA}+\mathrm{OxP} \rightarrow \mathrm{Glu}+\mathrm{RdP}$

v50: $\mathrm{His} \rightarrow \mathrm{Glu}+\mathrm{NH}_{4}^{+}$

v51: $\operatorname{Asp}_{\text {ext }} \rightarrow$ Asp

v52: $\mathrm{Cys}_{\text {ext }} \rightarrow \mathrm{Cys}$

v53: Gly $\rightarrow$ Gly $_{\text {ext }}$

v54: $\mathrm{Ser}_{\text {ext }} \rightarrow \mathrm{Ser}$

v55: Glu ext $_{\rightarrow} \mathrm{Glu}$

v56: $\mathrm{Tyr}_{e x t} \rightarrow \mathrm{Tyr}$

v57: Ala $\rightarrow$ Ala $_{\text {ext }}$

Protein Synthesis

v58:0.023 His +0.053Ile +0.091 Leu + 0.059 Lys + 0.023Met + 0.039Phe + $0.059 \mathrm{Thr}+0.014 \mathrm{Trp}$

$+0.066 \mathrm{Val}+0.051 \mathrm{Arg}+0.019 \mathrm{Cys}+0.042 \mathrm{Gln}+0.072 \mathrm{Gly}+0.052$ Pro+ $0.032 \mathrm{Tyr}+0.78 \mathrm{Ala}$

$+0.043 \mathrm{Asn}+0.053 \mathrm{Asp}+0.063 \mathrm{Glu}+0.068 \mathrm{Ser}+3 \mathrm{ATP} \rightarrow$ Protein + AMP + $\mathrm{Pp}_{i}+2 \mathrm{ADP}+2 \mathrm{P}_{i}$

Nucleotide Synthesis

v59: R5P + ATP $\rightarrow$ PRPP + AMP

v60: $\mathrm{PRPP}+2 \mathrm{Gln}+\mathrm{Asp}+\mathrm{Gly}+4 \mathrm{ATP}+\mathrm{CO}_{2} \rightarrow \mathrm{IMP}+2 \mathrm{Glu}+\mathrm{Fum}+4$

$\mathrm{ADP}+4 \mathrm{P}_{i}+\mathrm{Pp}_{i}$

$\mathrm{v} 61: \mathrm{IMP}+\mathrm{Asp}+3 \mathrm{ATP} \rightarrow \mathrm{ATP}_{R N}+\mathrm{Fum}+3 \mathrm{ADP}+\mathrm{P}_{i}$

v62: $\mathrm{IMP}+\mathrm{Gln}+3 \mathrm{ATP}+\mathrm{OxP} \rightarrow \mathrm{GTP}_{R N}+\mathrm{Glu}+2 \mathrm{ADP}+\mathrm{AMP}+\mathrm{Pp}_{i}+$ $\mathrm{RdP}$

v63: $\mathrm{CO}_{2}+\mathrm{NH}_{4}^{+}+\mathrm{Asp}+2 \mathrm{ATP}+\mathrm{OxP} \rightarrow$ Orotate $+\mathrm{RdP}+2 \mathrm{ADP}+2 \mathrm{P}_{i}$

v64: Orotate $+\mathrm{PRPP}+\mathrm{ATP} \rightarrow \mathrm{UTP}_{R N}+\mathrm{CO}_{2}+2 \mathrm{ADP}+\mathrm{Ppi}$

v65: $\mathrm{UTP}_{R N}+\mathrm{Gln}+\mathrm{ATP} \rightarrow \mathrm{CTP}_{R N}+\mathrm{Glu}+\mathrm{ADP}+\mathrm{P}_{i}$

v66: $0.285 \mathrm{ATP}_{R N}+0.285 \mathrm{UTP}_{R N}+0.215 \mathrm{GTP}_{R N}+0.215 \mathrm{CTP}_{R N} \rightarrow \mathrm{RNA}$

v67: $\mathrm{ATP}_{R N} \rightarrow \mathrm{dATP}$

v68: $\mathrm{GTP}_{R N} \rightarrow \mathrm{dGTP}$

v69: $\mathrm{UTP}_{R N} \rightarrow \mathrm{dTTP}$

v70: $\mathrm{CTP}_{R N} \rightarrow \mathrm{dCTP}$

v71: $0.285 \mathrm{dATP}+0.285 \mathrm{dTTP}+0.215 \mathrm{dGTP}+0.215 \mathrm{dCTP} \rightarrow$ DNA

Lipid Synthesis

v72: $\mathrm{DHAP}+\mathrm{RdP} \rightarrow$ Glyc3P $+\mathrm{OxP}$

v73: Glyc3P $+18 \mathrm{AcCoA}+21 \mathrm{ATP}+33 \mathrm{RdP} \rightarrow \mathrm{PA}+16\left(\mathrm{ADP}+\mathrm{P}_{i}\right)+33$

$\mathrm{OxP}+5\left(\mathrm{AMP}+\mathrm{Pp}_{i}\right)$

v74: $\mathrm{PA} \rightarrow(1,2) \mathrm{DG}+\mathrm{P}_{i}$ 
v75: $\mathrm{Eth}+(1,2) \mathrm{DG}+2 \mathrm{ATP} \rightarrow \mathrm{PE}+\mathrm{ADP}+\mathrm{P}_{i}+\mathrm{AMP}+\mathrm{Pp}_{i}$

v76: Chol $+1,2) \mathrm{DG}+2 \mathrm{ATP} \rightarrow \mathrm{PC}+\mathrm{ADP}+\mathrm{P}_{i}+\mathrm{AMP}+\mathrm{Pp}_{i}$

v77: $\mathrm{PE}+\mathrm{Ser} \rightarrow \mathrm{PS}+\mathrm{Eth}$

v78: $8 \mathrm{AcCoA}+8 \mathrm{ATP}+15 \mathrm{RdP}+\mathrm{Ser} \rightarrow \mathrm{Sphg}+7\left(\mathrm{ADP}+\mathrm{P}_{i}\right)+15 \mathrm{OxP}$

$+\mathrm{CO}_{2}+\mathrm{AMP}+\mathrm{Pp}_{i}$

v79: $\mathrm{Sphg}+8 \mathrm{AcCoA}+8 \mathrm{ATP}+14 \mathrm{RdP} \rightarrow \mathrm{Cer}+7\left(\mathrm{ADP}+\mathrm{P}_{i}\right)+14 \mathrm{OxP}$

$+\mathrm{AMP}+\mathrm{Pp}_{i}$

v80: $\mathrm{Cer}+\mathrm{PC} \rightarrow \mathrm{SM}+(1,2) \mathrm{DG}$

v81: 6 AcetoAcCoA $+6 \mathrm{AcCoA}+18 \mathrm{ATP}+14 \mathrm{RdP} \rightarrow$ Cholesterol +14

$\mathrm{OxP}+18 \mathrm{ADP}+4 \mathrm{Pp}_{i}$

$+6 \mathrm{P}_{i}+6 \mathrm{CO}_{2}$

v82: $0.5 \mathrm{PC}+0.2 \mathrm{PE}+0.075 \mathrm{PS}+0.075 \mathrm{SM}+0.15$ Cholesterol $\rightarrow$ Membrane Lipid 


\begin{tabular}{ll|ll|ll|ll|ll} 
v1 & 0.18713 & $\mathrm{v} 21$ & 0.01570 & $\mathrm{v} 41$ & 0.00849 & $\mathrm{v} 61$ & 0.00687 & $\mathrm{v} 81$ & 0.00025 \\
v2 & 0.14891 & $\mathrm{v} 22$ & 0.00000 & $\mathrm{v} 42$ & 0.00068 & $\mathrm{v} 62$ & 0.00518 & $\mathrm{v} 82$ & 0.00167 \\
v3 & 0.14762 & $\mathrm{v} 23$ & 0.00000 & $\mathrm{v} 43$ & 0.00068 & $\mathrm{v} 63$ & 0.01205 & & \\
v4 & 0.29653 & $\mathrm{v} 24$ & 0.04879 & $\mathrm{v} 44$ & 0.00157 & $\mathrm{v} 64$ & 0.01205 & & \\
v5 & 0.24774 & $\mathrm{v} 25$ & 0.01440 & $\mathrm{v} 45$ & 0.00120 & $\mathrm{v} 65$ & 0.00518 & & \\
v6 & 0.00000 & $\mathrm{v} 26$ & 0.03426 & $\mathrm{v} 46$ & 0.00205 & $\mathrm{v} 66$ & 0.00000 & & \\
v7 & 0.00000 & $\mathrm{v} 27$ & 0.00108 & $\mathrm{v} 47$ & 0.00205 & $\mathrm{v} 67$ & 0.00687 & & \\
v8 & 0.00000 & $\mathrm{v} 28$ & 0.00176 & $\mathrm{v} 48$ & 0.00205 & $\mathrm{v} 68$ & 0.00518 & & \\
v9 & 0.06698 & $\mathrm{v} 29$ & 0.00504 & $\mathrm{v} 49$ & 0.00410 & $\mathrm{v} 69$ & 0.00687 & & \\
v10 & 0.06109 & $\mathrm{v} 30$ & 0.00202 & $\mathrm{v} 50$ & 0.00326 & $\mathrm{v} 70$ & 0.00518 & & \\
v11 & 0.07202 & $\mathrm{v} 31$ & 0.00202 & $\mathrm{v} 51$ & 0.00023 & $\mathrm{v} 71$ & 0.02411 & & \\
v12 & 0.09943 & $\mathrm{v} 32$ & 0.01145 & $\mathrm{v} 52$ & 0.00089 & $\mathrm{v} 72$ & 0.00129 & & \\
v13 & 0.02953 & $\mathrm{v} 33$ & 0.00184 & $\mathrm{v} 53$ & 0.00235 & $\mathrm{v} 73$ & 0.00129 & & \\
v14 & 0.34451 & $\mathrm{v} 34$ & 0.00184 & $\mathrm{v} 54$ & 0.00013 & $\mathrm{v} 74$ & 0.00129 & & \\
v15 & 0.00894 & $\mathrm{v} 35$ & 0.00144 & $\mathrm{v} 55$ & 0.00944 & $\mathrm{v} 75$ & 0.00046 & & \\
v16 & 0.10879 & $\mathrm{v} 36$ & 0.00144 & $\mathrm{v} 56$ & 0.00755 & $\mathrm{v} 76$ & 0.00096 & & \\
v17 & 0.02823 & $\mathrm{v} 37$ & 0.00244 & $\mathrm{v} 57$ & 0.00881 & $\mathrm{v} 77$ & 0.00013 & & \\
v18 & 0.06988 & $\mathrm{v} 38$ & 0.00244 & $\mathrm{v} 58$ & 0.00173 & $\mathrm{v} 78$ & 0.00013 & & \\
v19 & 0.00000 & $\mathrm{v} 39$ & 0.01093 & $\mathrm{v} 59$ & 0.02411 & $\mathrm{v} 79$ & 0.00013 & & \\
v20 & 0.02955 & $\mathrm{v} 40$ & 0.00093 & $\mathrm{v} 60$ & 0.01205 & $\mathrm{v} 80$ & 0.00013 & &
\end{tabular}

Table 2: A vector $\mathbf{v}$ of admissible metabolic flux rates $\left(\mathrm{mM} /\left(\mathrm{h} \times 10^{9}\right.\right.$ cells $\left.)\right)$ consistent with the metabolic network and the experimental data of Table 1. 


\begin{tabular}{|c|c|}
\hline స్ & $00000000000000000 \stackrel{\mathscr{N}_{\mathrm{N}}}{0} 000000000$ \\
\hline ה & 000000000000000000000000000 \\
\hline & 000000000000000000000000000 \\
\hline 0 & 000000000000000000000000000 \\
\hline$\stackrel{\infty}{\infty}$ & 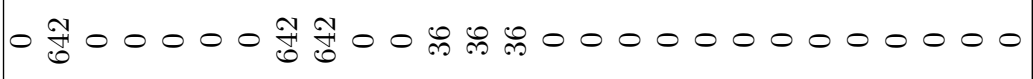 \\
\hline 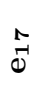 & $00000000000000000000000 \stackrel{n}{n}^{n} 000$ \\
\hline ब) & 000000000000000000000000000 \\
\hline d & 000000000000000000000000000 \\
\hline$\underset{J}{J}$ & 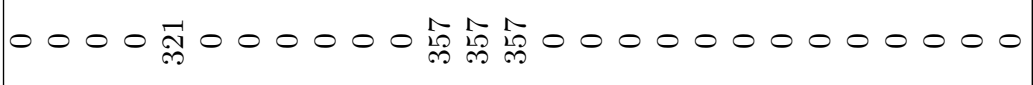 \\
\hline$\stackrel{m}{\dot{\theta}}$ & 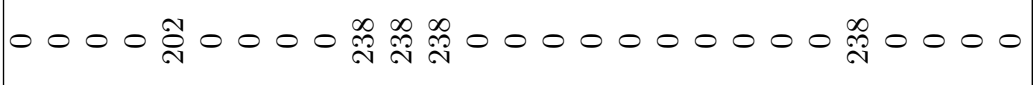 \\
\hline$\stackrel{N}{\mathcal{O}}$ & 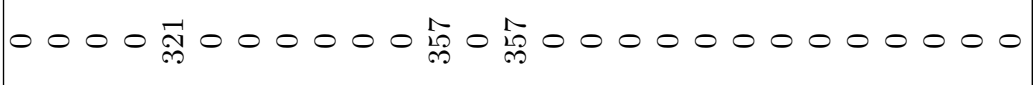 \\
\hline$\vec{J}$ & 00 苫 \\
\hline$\stackrel{2}{\circ}$ & 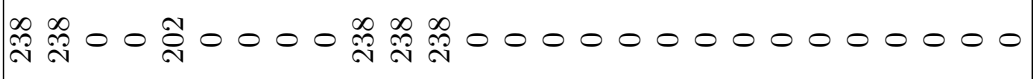 \\
\hline 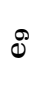 & 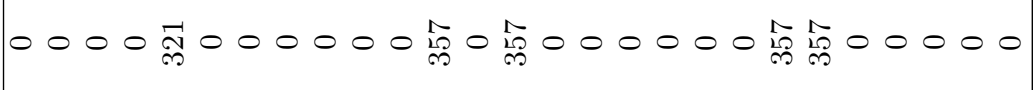 \\
\hline$\stackrel{\infty}{\infty}^{\infty}$ & 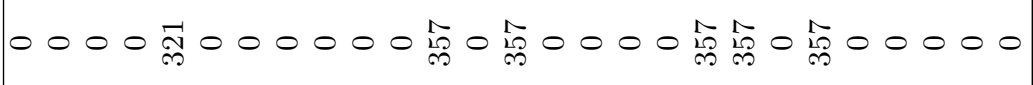 \\
\hline$\hat{\jmath}$ & 000000000000000000000000000 \\
\hline$\ddot{0}$ & 000000000000000000000000000 \\
\hline $\mathscr{0}$ & 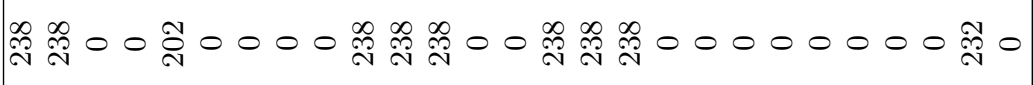 \\
\hline$\vec{J}^{\vec{\prime}}$ & $0000000000000000 m 0000000 m 00$ \\
\hline $\mathscr{\mathcal { O }}$ & 000000000000000000000000000 \\
\hline ঠ & 000000000000000000000000000 \\
\hline$\vec{b}$ & 00 苫 \\
\hline & 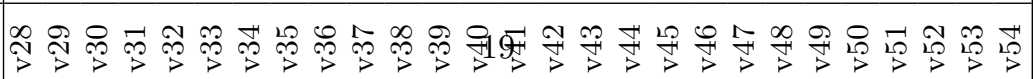 \\
\hline
\end{tabular}

Table 4: Elementary vectors $\mathbf{e}_{i}$ (entries 28 to 54 ) of the minimal decomposition of the flux vector $\mathbf{v}$ of Table 2 . The integer entries are exact stoichiometric coefficients. The other entries are truncated to the 2 nd decimal. 


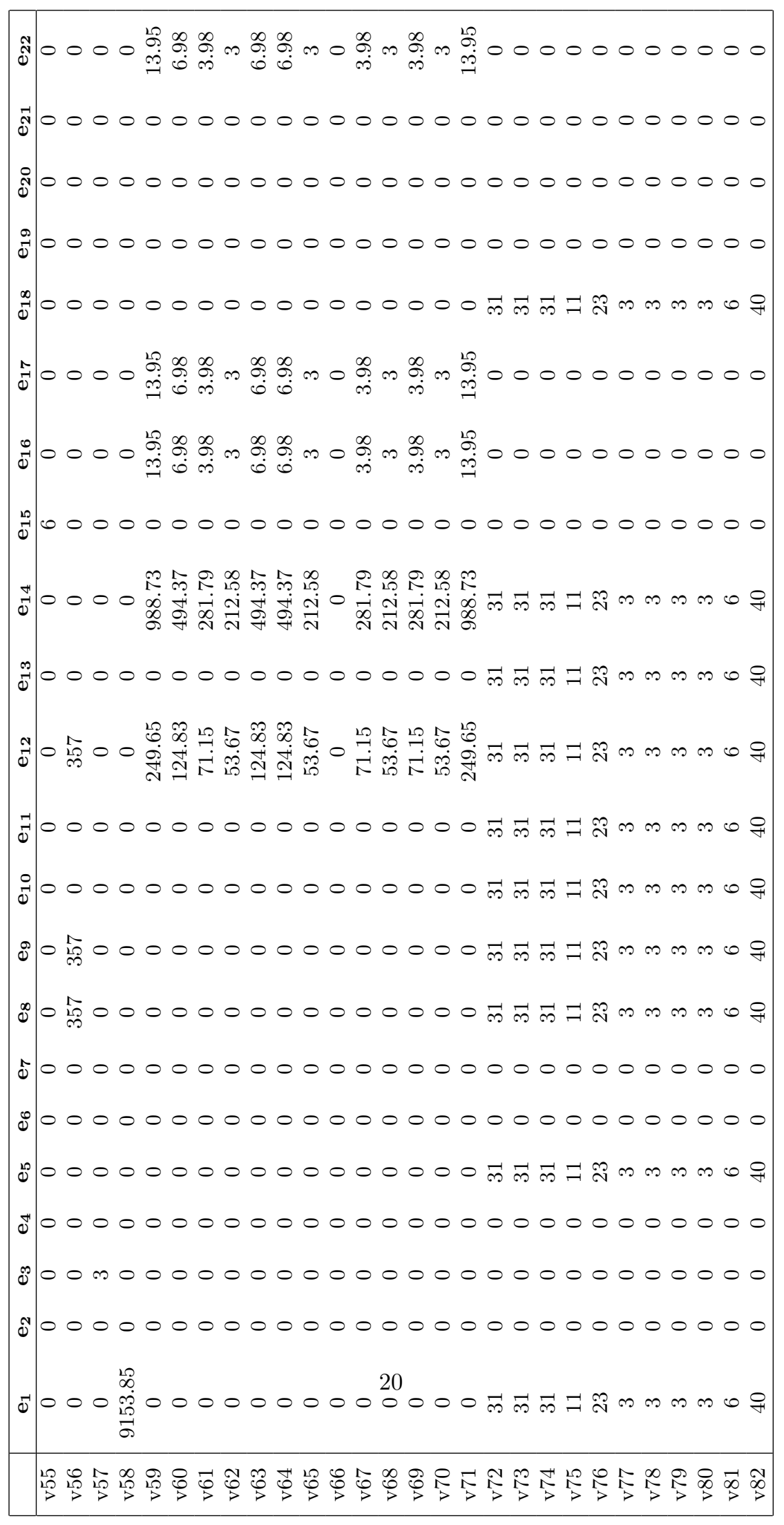

Table 5: Elementary vectors $\mathbf{e}_{i}$ (entries 55 to 82 ) of the minimal decomposition 